\title{
Berufsbildung im Gesundheitswesen: Ein Sonderweg mit Fragezeichen
}

\author{
Das Berufsbildungssystem im Gesundheitswesen geht einen Sonderweg. In traditionellen \\ Kernberufen wie der Pflege wird es von Länder- und Bundesministerien reguliert und \\ nicht wie sonst üblich nach dem Ordnungsrahmen des dualen Systems. Während unter \\ dem staatlichen Dach die Weiterentwicklung wichtiger Gesundheitsberufe nur schlep- \\ pend vorankommt, findet sich in solchen gesundheitsbezogenen Berufsfeldern, die nicht \\ der öffentlichen Zuständigkeit unterliegen, ein dynamisches, aber strategisch wenig fun- \\ diertes „Berufebasteln“. Die Konsequenzen sind eine unübersichtliche Aus- und Weiter- \\ bildungslandschaft - häufig mit Abschlüssen, deren Zukunftsfähigkeit fraglich ist. Der \\ Ruf nach einem Ende des Sonderwegs kann von daher nicht überraschen.
}

JOSEF HILBERT, CHRISTOPH BRÄUTIGAM, MICHAELA EVANS

\section{Einleitung}

Das System der dualen Berufsbildung wird als eine große Stärke des Standorts Deutschland gesehen. Die Bundesregierung engagiert sich zunehmend, dieses System - zumindest in seinen Grundorientierungen - anderen Ländern zu empfehlen und zu erläutern. Der Terminus „Facharbeit“" zielt auf eine breite, aber fachlich klar umrissene, praxisnahe und unter starker Praxisbeteiligung vermittelte Qualifikation. Sie orientiert sich an einer vollen beruflichen Handlungsfähigkeit, was die persönliche Disposition zur selbstständigen Planung, Durchführung und Kontrolle beruflicher Aufgaben einschließt. Facharbeit gilt als Rückgrat wirtschaftlicher Leistungsfähigkeit, Wettbewerbsfähigkeit und Beschäftigung.

Das deutsche Gesundheitswesen, einer der größten Wirtschaftszweige und Ausbildungsplatzanbieter, geht in der beruflichen Bildung einen Sonderweg. Auch hier spielt Facharbeit eine entscheidende Rolle, jedoch gelten die Vorgaben des Berufsbildungsgesetzes (BBiG) nur für einige wenige Berufsbilder, während der Löwenanteil der Ausbildungsplätze - vor allem in der Alten- und Gesundheits- sowie (Kinder)Krankenpflege - durch Berufsgesetze geregelt ist und durch eigenständige Institutionen und Verfahren verwaltet wird. Während das duale System durch ein elaboriertes Zusammenspiel von staatlichen Stellen, Arbeitgeberverbänden, Kammern und Gewerk- schaften reguliert und gesteuert wird, liegen die entsprechenden Kompetenzen und Zuständigkeiten im Gesundheitswesen weitgehend beim Staat. Verantwortlich sind die Ministerien für Gesundheit und Soziales, die sich im föderalen Kontext einerseits zwischen den Ländern untereinander und andererseits zwischen der Bundes- und der Länderebene unter anderem auf Ausbildungsziele, -inhalte, -dauer sowie auf Ausbildungswege verständigen müssen.

Der nachfolgende Beitrag setzt sich mit den Herausforderungen für die aktuelle und zukünftige Facharbeit im Gesundheitswesen auseinander. Er schildert, welche Umbrüche zu beobachten sind und welche Herausforderungen für das Gestaltungsfeld „Arbeit und Qualifizierung" entstehen (Abschnitt 2 und 3). Thematisiert werden zunächst die Reaktionen der Bildungsanbieter auf die Umbrüche und Erneuerungen. Empirische Daten zur Beschäftigtensicht auf das Zusammenspiel von Arbeitsgestaltung und Qualifizierung im Krankenhaus ergänzen die Darstellung (4). Im nächsten Schritt wird erörtert, ob und wie eine Orientierung an Prinzipien des dualen Systems dem Gesundheitssektor Vorteile bringen könnte (5). Abschließend wird vorgestellt, welche ausgewählten Gestaltungsprinzipien des dualen Systems auch für die Gesundheitsberufe nutzbringend sein können (6). 


\section{Gesundheitswirtschaft: arbeitspoli- tisch am Scheideweg?}

Die Gesundheitswirtschaft ist eine der größten Wirtschaftsbranchen in Deutschland. Hier arbeiteten im Jahr 2012 nahezu 6 Mio. Erwerbstätige, was rund 14,5 \% der Gesamtbeschäftigung in Deutschland entspricht (BMWi 2013). Im Jahr 2011 wurden von den knapp 1,7 Mio. Auszubildenden in Deutschland insgesamt gut 226.000 - also mehr als $13 \%$ - in einem Gesundheitsberuf ausgebildet (BIBB 2013, S. 27). Mit knapp 260 Mrd. € realisiert die Gesundheitswirtschaft derzeit einen Anteil von 11,1 \% an der Bruttowertschöpfung der Gesamtwirtschaft. Über die Hälfte der Wertschöpfung wird in den klassischen Bereichen der ambulanten und stationären Versorgung (inklusive Altenhilfe) erwirtschaftet - für die traditionell auch der Begriff "Gesundheitswesen“ verwendet wird.

Im Gesundheitswesen finden seit Jahren erhebliche Umbrüche statt, die in ihrer Vielfalt und Tiefe hier nur kurz skizziert werden: ${ }^{1}$ Das Altern der Bevölkerung, neue wissenschaftliche Erkenntnisse und Möglichkeiten sowie ein wachsendes Gesundheitsbewusstsein lassen den Bedarf und die Nachfrage nach gesundheitsbezogenen Dienstleistungen und Produkten deutlich steigen. In allen Bereichen des Gesundheitswesens sind Anstrengungen notwendig, die vorhandenen Mittel effizienter zu nutzen. Die Budgetierung der zur Verfügung stehenden Mittel, insbesondere die Einführung der Finanzierung nach Fallpauschalen im Krankenhaus, hat einen starken Kosten-, Effizienz- und Qualitätsdruck ausgelöst. Dieser beruht auch auf einem wachsenden Wettbewerb der Gesundheitsanbieter untereinander, darunter in den letzten zwei Jahrzehnten zunehmend auch private Anbieter. Mit der Einführung der Pflegeversicherung wurde in der Altenhilfe die Finanzierung für den einzelnen Pflegebedürftigen gedeckelt, der Kostendruck erhöht und der Wettbewerb forciert. Dies löste ähnliche Effekte aus wie die Einführung von Fallpauschalen im Krankenhausbereich. Gleichzeitig steigen die Anforderungen an die Integration der gesundheitlichen Versorgung. Sowohl seitens der Patienten und der Gesundheitspolitik als auch durch weitsichtige Anbieter wird darauf gedrängt, die gesundheitliche Versorgung über die Grenzen von Institutionen (ambulant und stationär) wie über die Grenzen medizinischer Disziplinen hinweg integriert und patientenorientiert zu gestalten. Für die Zukunft ist mit vielfältigen Innovationen zu rechnen. Es eröffnen sich neue Möglichkeiten der Gesunderhaltung, Diagnostik und Heilung, aber auch neue Perspektiven für eine Reorganisation von Versorgungsprozessen.

Der Umgang mit diesen Umbrüchen verlangt von den Krankenhäusern, Pflegeeinrichtungen, Arztpraxen und sonstigen Gesundheitseinrichtungen weitreichende Anpassungsschritte. In personalintensiven Branchen wie dem Gesundheitswesen können diese nicht ohne gravierende Erneuerungen beim Arbeitseinsatz und bei der Qualifizie- rung erfolgen. Noch gibt es keinen Kanon bewährter Maßnahmen, an dem sich Arbeitgeber, Beschäftigte und ihre Interessenvertreter orientieren können. Ein Blick in die Programme und die einschlägigen Beiträge der im Gesundheitssektor laufenden Zukunftskongresse sowie eine Sichtung einschlägiger empirischer Bestandsaufnahmen - etwa des „Innovationspanels Krankenhäuser“ (Blum et al. 2012a), des Krankenhausbarometers (Blum et al. 2012b), der „Landesberichterstattung Gesundheitsberufe NRW“ (Isfort et al. 2012) sowie des „Arbeitsreports Krankenhaus“ (Bräutigam et al. 2013a) - erlauben dennoch eine Übersicht über wichtige Reaktionsmuster.

In nahezu allen empirischen Erhebungen wird deutlich, dass in den letzten Jahren in Krankenhäusern und Pflegeeinrichtungen Arbeitsverdichtung an der Tagesordnung war. Gleichzeitig ist die Einkommensentwicklung für große Teile des Personals - vor allem für eher gering qualifizierte Helfer- und Assistenzberufe - eher unter dem Durchschnitt der deutschen Wirtschaft verlaufen (Bellmann et al. 2013; Evans et al. 2013). Parallel dazu wurde zwar Beschäftigung ausgebaut, jedoch wurde dieses Wachstum vorwiegend durch einen Zuwachs bei den Teilzeitstellen getragen. Als Folge des wirtschaftlichen Drucks und ermöglicht durch die arbeitsrechtlichen Deregulierungen sowie die Arbeitsmarktreformen zwischen 2002 und 2004 war auch ein Ausbau atypischer Beschäftigung und solcher mit geringen Qualifikationsanforderungen zu verzeichnen (Bellmann et al. 2013; Oschmiansky 2013, S. 220ff.). Allerdings wuchs die geringfügige Beschäftigung im Gesundheitswesen insgesamt etwas weniger als im Durchschnitt der Gesamtwirtschaft. Als weitere Reaktion ist die Werbung und Ansprache zusätzlicher Arbeitskräfte zu nennen: In den letzten Jahren wurden zum einen Imagekampagnen für Gesundheitsberufe auf den Weg gebracht, die von Anbietern und Gemeinschaftsinitiativen getragen wurden. Zum anderen - und dies ist eine jüngere Entwicklung etwa seit 2011 - wird im Ausland um Mediziner und Pflegekräfte geworben.

Das Krankenhausmanagement konzentriert sich insbesondere darauf, mit umgestalteten Organisationsstrukturen auf die sich wandelnden Anforderungen zu reagieren. Darüber hinaus ist an vielen Stellen ein aktiveres Personalmanagement zu beobachten, was insbesondere den Ausbau der Kommunikation zu den Beschäftigten vorantreibt etwa durch Mitarbeiterversammlungen, Intranet oder auch durch Mailings (DKG 2013, S. 17ff.). Verbesserungen der Arbeitsgestaltung dagegen werden eher zögerlich umgesetzt. Aus einer Online-Befragung von Beschäftigten in Krankenhäusern (Bräutigam et al. 2013a) geht hervor, dass die Beschäftigten in den letzten Jahren einen deutlichen Anstieg ihrer Verantwortung wahrnehmen. Zwar fühlen sie sich

1 Als Ein- und Überblicke zu diesen Themen siehe unter anderen Gerlinger (2013) sowie die verschiedenen Beiträge in Goldschmidt/Hilbert (2009); Lohmann/Preusker (2013); Mühlbauer et al. (2012). 
dafür mit ihren Qualifikationen fachlich einigermaßen gerüstet, berichten jedoch über schlechte Arbeitsbedingungen und darüber, ihre Arbeitsplätze nicht stärker im Sinne der steigenden Verantwortung mitgestalten zu können.

Die Tariflandschaft und die Verhandlungssysteme in der Gesundheits- und Sozialwirtschaft sind überaus kleinteilig und fragmentiert strukturiert (Evans et al. 2013; Bispinck et al. 2012; vgl. auch Dahme et al. 2012). Dies erschwert branchenweite Abschlüsse mit grundlegenden Verbesserungen. Darüber hinaus arbeiten Teile der Branche ohne Tarifbindung sowie mit Outsourcing und Leiharbeit.

Insgesamt stellt sich das Gesundheitswesen als ein bedeutender Wirtschaftszweig dar, der in den letzten Jahren gewachsen ist. Auch für die Zukunft sind weitere Umsatzund Beschäftigungsgewinne wahrscheinlich. Allerdings steht die Branche gleichzeitig vor enormen Anpassungsund Erneuerungsherausforderungen. Welche Rolle Facharbeit bei der Bewältigung der Herausforderungen spielen wird, ist noch nicht in allen Einzelheiten erkennbar. Allerdings zeigen empirische Untersuchungen, dass der Branche bislang die Vision, die Konzepte, die Gestaltungsinstrumente wie auch die Ressourcen für eine umfassende, sozialinnovative Aufwertung von Arbeit fehlen. Von einer systematischen Gestaltungsoffensive in Richtung gleichermaßen attraktiverer und produktiverer Arbeitsplätze mit höherer Qualifizierung, einem verbesserten Zusammenspiel von Arbeit und Technik sowie interaktiven Dienstleistungen mit Patienten (Dunkel/Weihrich 2012) kann bislang keine Rede sein. Auch wird an vielen Stellen mit „billiger Arbeit“ experimentiert. Damit zeigen sich Trends, die aus vielen anderen Branchen ebenfalls bekannt sind: steigende Verantwortung bei zunehmender Arbeitsverdichtung und einem gleichzeitigen Vormarsch atypischer Beschäftigung. Bosch/ Weinkopf (2011) bezeichnen vor allem den Dienstleistungssektor als „Experimentierfeld für prekäre Arbeit" - dies gilt auch im Gesundheitswesen.

\section{Berufsbildung: Unübersichtlichkeit und „Berufebasteln“}

Das Gesundheitswesen ist geprägt durch eine große, engagierte, äußerst vielfältige und sich dynamisch (oder sogar turbulent) verändernde Qualifizierungslandschaft. Für Insider und erst recht für Außenstehende erscheint sie oft unübersichtlich und an vielen Stellen fragmentiert. ${ }^{2}$ In einer Studie über die Entwicklung der Berufsbildung im Krankenhaussektor für die Friedrich-Ebert-Stiftung (FES) und die Vereinte Dienstleistungsgewerkschaft ver.di (vgl. Bräutigam et al. 2013a) wurde die Qualifizierungslandschaft für Gesundheitsberufe wie folgt umrissen:

Bislang gibt es keine verbindlichen und allgemeingültigen Definitions- und Qualitätsmerkmale für „Gesundheitsberufe“. Dementsprechend ist nicht nur die Verwendung des Begriffs vielfältig, sondern auch die Qualifizierungsangebote sind breit gefächert. Das Spektrum der fallweise berücksichtigten „Berufe" reicht von 36 Gesundheitsfachberufen im engeren Sinne (Isfort et al. 2012) über $800 \mathrm{Ge-}$ sundheitsberufe „in der äußerst dynamischen Gesundheitsbranche“ (Hamburger Abendblatt, 31.1.2009) bis zu 1.900 gesundheitswirtschaftlichen "Berufsangeboten“ in einem weiter gefassten Verständnis (www.gesundheitsberufe.de). Bei den Gesundheitsfachberufen im engeren Sinne wird unterschieden zwischen den akademischen Heilberufen (zum Beispiel Ärzte, Psychotherapeuten, Apotheker) und nicht-akademischen Fachberufen des Gesundheitswesens wie Gesundheits- und (Kinder)Krankenpflegern, Altenpflegern, Physiotherapeuten, Logopäden, Ergotherapeuten und Hebammen/Entbindungspflegern. Die Ausbildungen in diesen Fachberufen sind durch bundes- und landesrechtliche Regelungen strukturiert. Die wichtigsten sind das Krankenpflege-, das Altenpflege- sowie das Masseur-und Physiotherapeutengesetz. Nicht nur die Regelsetzung, auch die Umsetzung und Überwachung der Ausbildung sowie die Sorge um Finanzierungsregelungen sind in diesen Berufen in öffentlicher Zuständigkeit.

Im Anschluss an eine abgeschlossene Ausbildung in einem Gesundheitsfachberuf kann - insbesondere in der Pflege - eine große Zahl an Weiterbildungen absolviert werden. Dies sind zum einen die auf Länderebene geregelten Fachweiterbildungen zur Spezialisierung in verschiedenen klinischen Bereichen (etwa in der Anästhesie- und Intensivpflege oder zum Fachpfleger für Gerontopsychiatrie). Andere Weiterbildungen finden sich unter anderem im pädagogischen Bereich (Praxisanleitung) oder im Management (beispielsweise Leitung einer Station oder Einheit, Pflegedienstleitung). Ein Teil dieser Weiterbildungen wird mittlerweile zunehmend akademisiert. Dies gilt insbesondere für den pflegepädagogischen Bereich. Neben diesen Weiterbildungen gibt es vielfältige Fortbildungsangebote. Die Fortbildungen sind nicht landesrechtlich reguliert und werden von diversen Bildungsanbietern unterschiedlicher Trägerschaft zu einer Fülle von Themen wie „Gesprächsführung“ und „Umgang mit Konflikten“ bis hin zur Trauerbegleitung angeboten. An (Fach)Hochschulen können Absolventen der genannten Ausbildungen akademische Abschlüsse erwerben, zumeist unter der Voraussetzung, dass sie über die (Fach)Hochschulreife verfügen. Bekannt und etabliert sind Studiengänge wie Pflegewissenschaft, Pflegemanagement oder Pflegepädagogik. Seit einigen Jahren finden sich an ersten Hochschulen pflegewissenschaft-

2 Seit einigen Jahren erscheinen Veröffentlichungen mitTiteln wie "Gesundheitsberufe im Wandel" (etwa Bollinger et al. 2008 oder Höppner/Kuhlmey 2009), die allerdings immer nur Facetten des Wandels und kein Gesamtbild liefern. Das Internetportal www.gesundheitsberufe.de gibt zwar einen sehr faktenreichen Überblick, ist wegen der Vielfalt der Informationen aber auch recht unübersichtlich. 
liche Masterstudiengänge und Bachelorstudiengänge für das ursprünglich US-amerikanische „Advanced Nursing Practice“. Bei den Absolventen handelt es sich um „Pflegeexperten“ im Sinn klinisch tätiger Pflegeakademiker mit pflegerischer Berufsausbildung, deren Aufgaben klinikindividuell festgelegt werden.

Etwa seit Ende der 1980er Jahre gibt es bei den Pflegeberufen eine rege Reformdiskussion in Richtung einer generalistischen Pflegeausbildung, d.h., es geht um eine Zusammenführung der Gesundheits- und Krankenpflege, Gesundheits- und Kinderkrankenpflege sowie der Altenpflege, die mittlerweile auch zu positiv evaluierten Modellversuchen geführt hat. Eine grundlegende Reform in diese Richtung lässt jedoch noch immer auf sich warten (vgl. Abschnitt 5). Parallel zur nur äußerst langsam voranschreitenden Reform der Berufsausbildung in den Pflegeberufen erfolgten ein Einstieg und Ausbau grundständig berufsqualifizierender Studiengänge ${ }^{3}$ in der Pflege, dem Hebammenwesen und den therapeutischen Berufen Logopädie, Physiotherapie und Ergotherapie. Mittlerweile hat diese Perspektive die Unterstützung des Wissenschaftsrats (WR 2012).

Ein kleiner Teil der Gesundheitsberufe wird nicht von staatlichen Stellen allein, sondern im dualen System reguliert. Zu diesen Gesundheitsberufen gehören die Zahntechniker, die Augenoptiker, die Hörgeräteakustiker, die Orthopädiemechaniker, die Orthopädieschuhmacher sowie die Kauffrau bzw. der Kaufmann im Gesundheitswesen (Isfort et al. 2012).

Darüber hinaus entstanden auf Basis vorhandener Ausbildungen in den oben genannten nicht-akademischen $\mathrm{Ge}$ sundheitsfachberufen in den letzten Jahren vielfältige Spezialisierungen. Sie fokussieren entweder auf bestimmte Zielgruppen - etwa Patienten mit spezifischen Krankheitsbildern wie Schlaganfall oder Brustkrebs - oder auf spezifische Problemstellungen. Beispiele hierfür sind etwa „Stroke Nurses" oder „Breast Care Nurses" in der Pflege oder auch die sogenannten Fallmanager („Case Manager"). Rechtlich reguliert sind solche Spezialisierungsberufsbezeichnungen und deren Ausbildungen nicht, gleichwohl existieren anerkannte Zertifizierungsverfahren zum Beispiel durch Fachgesellschaften oder Verbände.

Immer mehr Verbreitung finden auch Berufsbezeichnungen, die auf „-assistent“ oder „-assistentin“ enden. Dahinter stecken Ausbildungen mit ganz unterschiedlicher Regulierung, Dauer und Qualifikation. Zum Ersten gibt es einige Assistenzberufe, die auf eine ein- bis zweijährige Ausbildung zurückgehen und öffentlich geregelt sind. Dies trifft etwa auf die Helferberufe in der Pflege zu. Zum Zweiten findet sich beispielsweise die Ausbildung zum medizinisch-technischen Assistenten bzw. zur Assistentin, eine Sammelbezeichnung für dreijährige Ausbildungen mit bundeseinheitlicher Regelung. Drittens werden einige neue akademische Abschlüsse mit dem Assistenz-Begriff beschrieben (zum Beispiel „Physican Assistant“, „Cardiology Assistant“). Zum Vierten werden mit dem Assistenz-Attri- but etliche kurze und betriebsinterne Qualifikationen bezeichnet, die auf Helfertätigkeiten zielen. Beispiele sind etwa Service-Assistenten oder Stations-Assistenten.

Auf Basis der im Kontext des Bologna-Prozesses gestiegenen Möglichkeiten der Hochschulen, Studiengänge selbst zu entwickeln und einzuführen, entstand eine Fülle von neuen Studiengängen mit Gesundheitsbezug. Die Palette der Master-Studiengänge reicht etwa vom „Master in Public Health“ (mit dem Schwerpunkt Psychosoziale Prävention und Gesundheitsförderung) über einen WeiterbildungsMaster in „Health Management" bis zum "Master in Naturheilkunde“. Der Bologna-Prozess bietet den (Fach) Hochschulen neue Chancen, die Gesundheitswirtschaft mit einer bis dato unbekannten Vielfalt von neuen Abschlüssen zu adressieren.

Auch die Finanzierung der beruflichen Bildung ist ähnlich vielfältig und unübersichtlich. Ein grober Überblick ergibt, dass Berufe, die einen engen Bezug zum Krankenhaus haben (vor allem Gesundheits- und (Kinder)Krankenpflege), überwiegend über Mittel aus den Krankenversicherungen getragen werden. Ausbildende Häuser erhalten sie in Form von Zuschlägen auf die Fallpauschalen, also die Erstattungen für die behandelten Krankheitsfälle. Im Bereich der Altenhilfe wird die praktische Ausbildung (inklusive der Ausbildungsvergütungen) von den ausbildenden Pflegeeinrichtungen oder - je nach Bundesland - über eine Umlage getragen, die sie über die Pflegesätze refinanzieren. Der schulische Teil der Ausbildung wird durch die Länder finanziert. Zum Teil wird dafür noch Schulgeld von den Auszubildenden verlangt. Bei vielen - gerade auch bei vielen neu entstandenen - Berufen und Ausbildungsgängen kommt es teilweise zu erheblichen (oder sogar zu 100 \%igen) Eigenbeiträgen der Auszubildenden. Bei Weiter-und Fortbildungen engagieren sich allerdings oft auch die Arbeitgeber und übernehmen die Qualifizierungskosten ganz oder teilweise.

Die vorstehende Skizze zeugt zum einen von der Vielfalt und Unübersichtlichkeit der Qualifizierungslandschaft. Zum anderen macht sie deutlich, dass es an einer strategischen Berufsbildungsplanung fehlt. Dort, wo staatliche Stellen dafür zuständig sind, wird diese Aufgabe zwar wahrgenommen, jedoch kommt es zu gravierenden Verzögerungen bei der Koordination von Ausbildungszielen, -inhalten, -dauer sowie der Ausbildungswege. Auch sind in den vergangenen Jahren Aufgaben zum Beispiel auf Akkreditierungsagenturen übertragen worden. In die Freiräume, die durch diesen faktischen „Regulierungsattentismus“ der öffentlichen Stellen entstehen, sind in den letzten Jahren zunehmend neue Anbieter mit neuen Angeboten gesprungen

3 Gemeint sind Studiengänge, die für die Berufstätigkeit qualifizieren und erstmals in Deutschland dem Nachwuchs in traditionell nicht-akademischen Berufen den Erwerb eines ersten akademischen Grades bereits mit der Berufsqualifikation ermöglichen. 
- von öffentlichen, freigemeinnützigen oder auch privaten (Fach)Hochschulen über Berufsakademien in unterschiedlicher Trägerschaft bis hin zu privaten Bildungskonzernen. Die berufliche Bildung im Gesundheitswesen wurde zum wichtigen Gestaltungs- und Expansionsfeld einer Bildungswirtschaft, die zwar dynamisch ist, deren Bemühungen allerdings teilweise an Aktionismus grenzen.

\section{Mismatch: Die Sicht der Beschäftig- ten}

Die Vielfalt, Unübersichtlichkeit und kaum erkennbare strategische Ausrichtung der beruflichen Bildung im Gesundheitswesen spiegelt sich in Befragungen der Beschäftigten in dieser Branche zu den Themen Arbeit und Qualifikation wider, wie etwa im „Arbeitsreport Krankenhaus“ (Bräutigam et al. 2013a). ${ }^{4}$ Als wichtige Ergebnisse können folgende Punkte genannt werden:

- Fachweiterbildung, Leitungsweiterbildung und pädagogische Weiterbildung sowie diverse pflegepraktische Fortund Weiterbildungen bestimmen das Qualifizierungsgeschehen in der Krankenhauspflege. Bisher ergänzend finden sich pflegebezogene Studiengänge sowie berufsbegleitende wissenschaftliche Weiterbildungen zum Beispiel in den Bereichen Gesundheitsökonomie oder Public Health. Die berufliche Nachqualifizierung zu einem (höherwertigen) Berufsabschluss spielt in den Einrichtungen hingegen kaum eine Rolle.

- Mehr als $56 \%$ der Befragten geben an, dass sie ihre vorhandenen Qualifikationen und Kompetenzen im Arbeitsalltag „vollkommen“ und „überwiegend“ einsetzen können. Demgegenüber gaben $32 \%$ der Befragten an, sie können ihre vorhandenen Qualifikationen und Kompetenzen nur „teilweise“ einsetzen. Knapp 12 \% können ihre vorhandenen Kompetenzen und Qualifikationen „weniger“ oder "gar nicht" einsetzen (Abbildung 1). Es scheint demzufolge Passungsprobleme zwischen Ausbildungs- und Qualifizierungsangeboten einerseits und dem konkreten Berufsalltag andererseits zu geben.

- Die Beschäftigen erwarten von einer Fort- und Weiterbildung nicht allein einen höheren Verdienst oder eine berufliche Neuorientierung, sondern maßgeblich auch eine bessere „Bewältigung der Arbeitsaufgaben“, die „Übernahme von mehr Verantwortung“ sowie die „Verbesserung der beruflichen Position (Aufstieg)“. Während die Ziele einer besseren Bewältigung von Arbeitsaufgaben sowie die Übernahme von Verantwortung vielfach erreicht werden, scheint sich die Verbesserung der beruflichen Position durch Bildungsmaßnahmen ebenso wie eine Erhöhung des Verdienstes wesentlich schwieriger zu gestalten.

- Fort- und Weiterbildung für die Beschäftigten werden seitens der Arbeitgeber zwar teilweise (mit)finanziert; der
ABB. 1

\section{Verwertbarkeit der Beschäftigtenqualifikationen im Krankenhaus-Arbeitsalltag*}

Angaben in Prozent

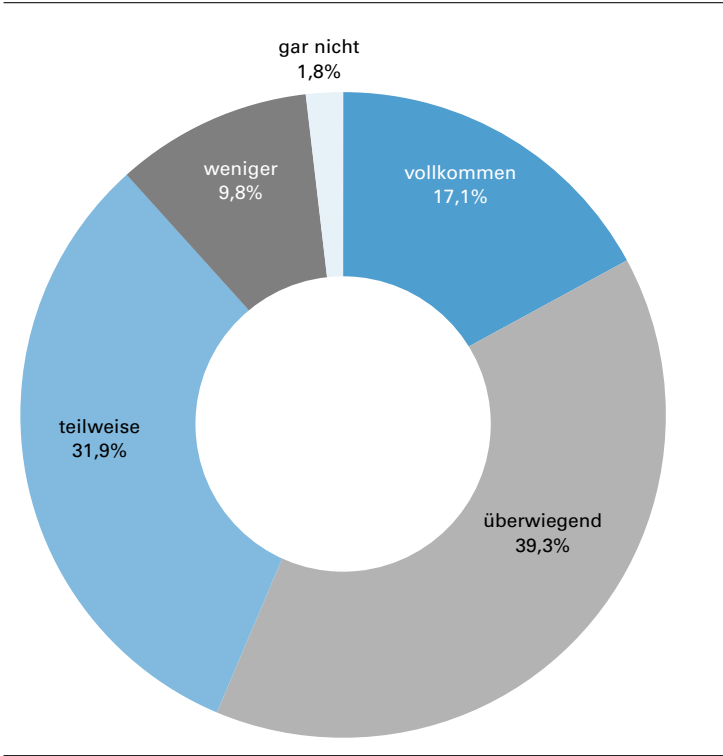

${ }^{*} n=3.404$

Quelle: Bräutigam et al. (2013a).

Mitteilungen

Bedarf hierfür wird jedoch nur selten systematisch erhoben, obwohl rund $73 \%$ der Befragten für die Zukunft einen weiteren Bedarf an Fort- und Weiterbildung sehen, um ihre Arbeitsaufgaben bewältigen zu können (ohne Abbildung). - Im Einsatzprofil der Pflege verändern sich die Aufgaben erheblich. Rund 78,6 \% der befragten Pflegenden haben in den vergangenen Jahren Aufgaben vom ärztlichen Dienst übernommen. Die Übernahme von Aufgaben erfolgte vielfach in einer Situation, in der gleichzeitig Pflegestellen abgebaut wurden.

- Wenngleich sich viele Krankenhäuser darum bemühen, die Patientenorientierung zu verbessern (etwa durch mehr Integration bei den Angeboten), sorgen sich viele Beschäftigte um die Versorgungsqualität bei den Patienten. So äußern lediglich knapp 15 \% der Befragten, dass die neue Aufgabenteilung zwischen Medizin, Pflege und Assistenzdiensten zu einer Verbesserung der Versorgungsqualität führt.

- Für die Zukunft sehen die Beschäftigten zwar die Notwendigkeit von Weiterbildung sowie der Übernahme

4 Unterstützt von der Hans-Böckler-Stiftung konnte das Institut Arbeit und Technik bis Februar 2013 bei einer OnlineBefragung 3.404 Beschäftigte aus Krankenhäusern zu den Themenblöcken Arbeit und Qualifizierung befragen. Diese Untersuchung ist zwar nicht repräsentativ, lässt aufgrund der hohen Teilnehmerzahl jedoch auf großes Interesse an den Themen der Befragung schließen. 
ABB. 2

\section{Arbeitsplatzbewertung der Beschäftigten im Krankenhaus \\ Angaben in Prozent \\ völlig zutreffend \\ ziemlich unzutreffend \\ ziemlich zutreffend \\ völlig unzutreffend}

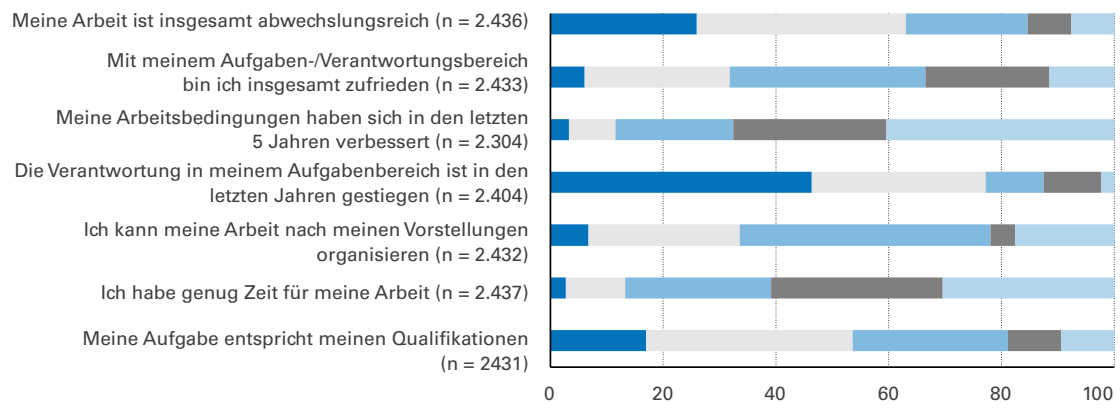

Quelle: Bräutigam et al. (2013a)

von mehr Verantwortung in ihrem Arbeitsbereich. Gleichzeitig wird jedoch auch deutlich, dass sie nicht mit mehr Entscheidungsspielräumen, einer besseren Vergütung oder einer besseren Nutzung ihrer erworbenen Qualifikationen rechnen (Abbildung 2).

Die vorstehend skizzierten empirischen Erkenntnisse aus dem „Arbeitsreport Krankenhaus“ über die Rolle von Qualifizierung und Arbeit aus Beschäftigtensicht sind an sich noch keine ausreichende Basis, um den Zustand und die Zukunftsfähigkeit des Systems der beruflichen Bildung im Gesundheitssektor detailliert bewerten zu können. Dazu wären weitergehende Erhebungen im Arbeitsalltag, bei $\mathrm{Ar}$ beitgebern und im Management, Befragungen von Patienten sowie internationale Vergleiche bzw. solche mit anderen Branchen erforderlich. Gleichwohl lassen die Befragungsergebnisse eine klare These zu: Die Vielpoligkeit, Unübersichtlichkeit, Geschäftigkeit und die fehlende strategische Fundierung in der Qualifizierungslandschaft treffen auf eine Arbeitswelt, die die Nutzung erworbener Qualifikationen erschwert. Darüber hinaus zeigen die Daten, dass die Beschäftigten mit der Fort- und Weiterbildung Ziele verbinden, die im Arbeitsalltag nur unzureichend eingelöst werden. $\mathrm{Ob}$ solche Passungsprobleme größer oder kleiner als in anderen Branchen und Sektoren der deutschen Wirtschaft sind, kann auf Basis der vorliegenden wissenschaftlichen Erkenntnisse nicht beurteilt werden. ${ }^{5}$ Ein Problem für die Attraktivität und für die Entwicklungsaussichten der Gesundheitsberufe sind sie gleichwohl.

In arbeitspolitischen Debatten wird derzeit oft die Befürchtung geäußert, dass es zu einer Erosion von Facharbeit und „Beruflichkeit“ kommen könnte. Gründe hierfür sind unter anderem defizitäre Infrastrukturen beruflicher Bildung im betrieblichen Kontext, eine unzureichende, kurz- fristige Planung und Organisation von Arbeitsprozessen, volatile Berufs- und Karrierewege sowie eine zunehmende Differenzierung von Beschäftigungsbedingungen innerhalb und zwischen Branchen (Voss-Dahm et al. 2011). In der Gesundheitswirtschaft finden sich vielfältige Anhaltspunkte für vergleichbare Entwicklungen und Befürchtungen.

\section{Duales System - Orientierung für den Gesundheitssektor?}

Die dargestellten Entwicklungen und Probleme haben bislang weder bei Unternehmen und Einrichtungen des Gesundheitswesens noch bei Interessenorganisationen zu einem massiven Ruf nach Umorientierung geführt. Erst recht fehlen gezielte Vorschläge für eine grundsätzliche Reform des Sonderwegs in der beruflichen Bildung. Lediglich aus Forschungs- und Diskussionszusammenhängen beim Bundesinstitut für Berufsbildung (BIBB) (vgl. u.a. Esser 2012), in engen Fachkreisen in der Vereinten Dienstleistungsgewerkschaft (ver.di) sowie aus dem Deutschen Industrie- und Handelskammertag (vgl. DIHK 2013) kamen vorsichtige Anregungen, sich in den Gesundheitsberufen stärker an den Leitprinzipien und Institutionen des dualen Systems zu orientieren. Auch der erste Gleichstellungsbericht der Bundesregierung empfiehlt: „Das in einen dualen und einen schulischen Zweig geteilte Berufsbildungssystem soll mit dem Ziel, bundeseinheitliche Standards in der Berufsausbildung zu schaffen, zusammengeführt und vereinheitlicht werden. “ (BMFSFJ 2011, S. 101)

Die duale Berufsausbildung in Deutschland stößt weltweit auf große Aufmerksamkeit und Anerkennung. Geregelt wird sie durch das Berufsbildungsgesetz (BBiG) beziehungsweise durch analoge Regelungen der Handwerksordnung (HWO). Faktisch ist sie vor allem dadurch geprägt, ${ }^{6}$ dass es quasi in allen Bereichen und Fragen der beruflichen Bildung - von der Planung und Abgrenzung von Berufsbildern über die Finanzierung bis hin zur Durchführung, Überwachung und Qualitätsentwicklung - zu einem systematisch organisierten und auf Konsens programmierten Zusammenspiel der zuständigen öffentlichen Stellen sowie der jeweiligen Arbeitgeberverbände, Kammern und Gewerkschaften kommt. Ein weiteres, von außen oft zuerst wahrgenommenes Merkmal ist darüber hinaus, dass die Ausbildung an zwei Standorten - in einer (Berufs)Schule und im Betrieb

5 Eine Übersicht zur Entwicklung der beruflichen Weiterbildung in Deutschland wurde etwa von Bosch (2012) vorgelegt.

6 Als Überblick siehe Euler (2013), die Beiträge in FES (2010) sowie den Beitrag von Bosch in diesem Heft. Zur korporatistischen Steuerung und Regulierung siehe Streeck et al. (1987). 
(bzw. ergänzend in überbetrieblichen praktischen Ausbildungsstätten) - stattfindet.

Innerhalb des angesprochenen Ordnungsrahmens gibt es eine Fülle von Detailregelungen, die hier nicht wiedergegeben werden können. Mit Blick auf Möglichkeiten, im Gesundheitssektor vom dualen System zu lernen, sind insbesondere Fragen der Strategieentwicklung sowie der Überwachung und Qualitätsentwicklung zu erwähnen. Bei der Strategieentwicklung fällt ins Auge, dass das Bundesinstitut für Berufsbildung (BIBB) als Kompetenzzentrum und als Dialogplattform für die an der Berufsbildung beteiligten Gruppen arbeitet und nicht zuletzt auch die Federführung für die Entwicklung von Ausbildungsordnungen wahrnimmt. Dabei verfügt das BIBB auch über Ressourcen und Verfahren, um wissenschaftliche Erkenntnisse einzuspeisen und für die Weiterentwicklung bestehender und die Entwicklung neuer Berufe fruchtbar zu machen.

Die Überwachung der Ausbildungsaktivitäten ,vor Ort “ - früher oft ein problematischer Punkt - wird von den Industrie- und Handelskammern bzw. den Handwerkskammern wahrgenommen. Gesteuert werden die einschlägigen Aktivitäten bei den Kammern von Berufsbildungsausschüssen, in denen die Gewerkschaften paritätisch vertreten sind. In den letzten Jahren gelang es zunehmend, die Überwachung im Sinne einer systematischen Qualitätsentwicklung $\mathrm{zu}$ interpretieren und auszubauen.

Die Wirkungen des dualen Systems für die Entwicklung des Arbeits- und Wirtschaftsstandorts Deutschland werden überwiegend positiv eingeschätzt. Mit Blick auf die oben skizzierten Probleme der beruflichen Bildung in der Gesundheitswirtschaft ist zunächst hervorzuheben, dass es mit den BBiG/HWO-Orientierungen und Verfahren gelingt, für mehr Vereinheitlichung und Transparenz zu sorgen. Dies schlug sich vor allem bei der Zahl der anerkannten Ausbildungsberufe nieder, die von 606 im Jahre 1971 auf 345 in 2012 sank (http://www.bibb.de/de/wlk26560.htm). Darüber hinaus kommt es im Rahmen des BBiG/HWO-Regelwerkes jeweils branchenweit zu einem systematisch angelegten und wissenschaftlich unterfütterten strategischen Austausch.

Welche Unterschiede zwischen den Gestaltungsverfahren bei den Gesundheitsfachberufen einerseits und den Berufen unter dem Dach des BBiG bestehen, ist bislang nicht systematisch erforscht. Anhaltspunkte für Einschätzungen und Hypothesen lassen sich aus den Neuregelungsbemühungen im Bereich der Pflegeberufe ableiten, in denen es darum geht, die verschiedenen Pflegeausbildungen (Gesundheitsund Krankenpflege, Gesundheits- und Kinderkrankenpflege, Altenpflege) zu einer einheitlichen Pflegeausbildung zu vereinigen (Bund-Länder Arbeitsgruppe 2012).

Die einschlägigen Aktivitäten bei der Reform der Pflegeausbildung (als Kurzüberblick siehe Dielmann 2013) gehen auf vielfältige Diskussionen seit Ende der 1980er Jahre zurück und wurden etwa um die Jahrtausendwende begonnen. Zwischen 2003 und 2008 führten sie zu einer Reihe von Modellversuchen, deren Auswertung die Machbarkeit einer solchen Integration stützte. Sie führten $2010 \mathrm{zu}$ einer
Bund-Länder-Arbeitsgruppe zur Weiterentwicklung der Pflegeberufe unter gemeinsamer Federführung des Bundesministeriums für Familie, Senioren, Frauen und Jugend (BMFSF) und des Bundesministeriums für Gesundheit (BMG), die 2012 Eckpunkte zur Vorbereitung eines neuen Pflegeberufsgesetzes vorlegte, dabei aber Finanzierungsfragen ausklammerte und nur noch als Darstellung möglicher Finanzierungsvarianten umriss (Bund-Länder-Arbeitsgruppe 2012). Ihre Arbeit wurde von einer schwer überschaubaren Fülle von Stellungnahmen - insbesondere aus der Welt der Fachverbände - begleitet. Noch ist offen, wann sie tatsächlich zu einem konkreten Gesetzgebungsverfahren führen wird.

Hinsichtlich der grundlegenden Regelungsaufgaben der Berufsbildungspolitik - vor allem Entscheidungen treffen, Finanzierungsregeln finden, Durchführung gewährleisten und überwachen - sind beim Vergleich zwischen dem skizzierten Fall der Neuordnung der Pflegeausbildung und den idealtypischen BBiG/HWO-Verfahren deutliche Unterschiede auszumachen. Sofort fällt auf, dass die Bemühungen um eine grundlegende Neuorientierung der Berufsbildung im Gesundheitswesen sich sehr lange hinziehen. Während die öffentlich verantwortete und öffentlich durchgeführte Erneuerung der Pflegeberufe offensichtlich im Dickicht des schwierigen Politikbetriebes nur sehr langsam vorankommt, funktionieren BBiG-Ordnungsverfahren zügiger - hier ist „Wandel trotz Reformstau“ (Busemeyer 2009) möglich. Becker/Meifort (2006) sehen eine der wichtigsten Ursachen für die Schwerfälligkeit bei der Pflegereform in der „,öderalen Zersplitterung“ der Zuständigkeiten.

Im Zusammenhang mit dem Neuordnungsverfahren zur Zusammenführung der Pflegeberufe wird des Weiteren deutlich, dass es weder auf Arbeitgeberseite noch auf Arbeitnehmerseite Instanzen gibt, die im Sinne einer Interessenvereinheitlichung wirken. Statt integrierter Stellungnahmen durch die jeweils zuständigen Interessenorganisationen und Gewerkschaften trifft Politik auf eine unübersichtliche Vielfalt an Analysen, Interessensäußerungen und Stellungnahmen. Während das BBiG Experten und Interessenvertreter von der Vielfalt zur Integration drängt, bietet die wegen der Zuständigkeit der unterschiedlichen Ministerien auf Bundes- und Länderebene komplizierte Lage bei der Pflege immer wieder neue Ansatzpunkte für Einwände, Bedenken und Infragestellungen. Das BBiG wirkt offensichtlich integrierender.

\section{Schlussfolgerung und Gestaltungs- vorschläge}

In Deutschland hat sich das duale System in seinen Grundzügen bewährt. Die Qualität des Ausbildungsgeschehens im Gesundheitssektor könnte von einer stärkeren Orientierung am BBiG profitieren. Eine Übertragung der Neu- 
ordnung der Gesundheitsberufe in das BBiG/HWO-Regelwerk ist allerdings schon allein deshalb unwahrscheinlich, weil viele der heute zuständigen und mitwirkenden Länder- und Bundesministerien nur widerstrebend Regelungszuständigkeiten abgeben würden.

Gleichwohl drängt sich die Empfehlung auf, dass sich interessierte Akteure aus der Gesundheitsbranche vermehrt an den Gestaltungsprinzipien des dualen Systems orientieren sollten. Prioritäre Ziele könnten dabei ein Zugewinn bei der Übersichtlichkeit sowie eine Schärfung der strategischen Orientierung - das heißt vor allem eine solide wissenschaftliche Fundierung sowie mehr Verständigungsdruck bei der Planung und Umsetzung neuer oder erneuerter Berufe - sein (vgl. Bräutigam et al. 2013b).

Mehr Übersichtlichkeit und Orientierung könnten mithilfe einer Plattform „Berufsbilder im Gesundheitssektor" geschaffen werden. Neben einer Übersicht über Berufsbilder und Bildungswege sollte sie insbesondere auch eine Einschätzung von Zukunftsaussichten neuer Berufsbilder sowie ein moderiertes Forum über Erfahrungen in einzelnen Berufen und Bildungswegen bieten.

Vor dem Hintergrund der oben umrissenen Turbulenzen und Unsicherheiten über die zukünftige Entwicklung der Arbeitswelt im Gesundheitssektor scheint darüber hinaus ein Ausbau der Forschung zur Lage und zur Entwicklung der Arbeit in den Gesundheitsberufen unerlässlich. Einem „Berufsbildungsbericht Gesundheitsberufe“ käme die Aufgabe zu, die verschiedenen Erkenntnisse im Sinne einer Metastudie zusammenzutragen, auszuwerten und zur Diskussion zu stellen. Dringend erforderlich wäre vor allem eine empirisch fundierte Quer- und Längsschnittberichterstattung zur Lage und zur Entwicklung von Qualifikationen, Tätigkeiten, Arbeitsroutinen und -bedingungen. ${ }^{7}$ Zusätzlich könnte die Branche von einem systematischen Trendmonitoring über innovative Gestaltungsmöglichkeiten im Zusammenspiel beruflicher Bildung und Arbeitsgestaltung profitieren. Entsprechende Vorarbeiten sind etwa mit dem „Arbeitsreport Krankenhaus“ oder der Veröffentlichung von Klaes et al. (2013) vorgelegt worden.

Sicherlich kann eine zukunftsträchtige Berufsbildungspolitik für den Gesundheitssektor nicht ohne ein starkes Engagement der öffentlichen Politik gelingen. Die oben angeregte stärkere Orientierung an zentralen Prinzipien des dualen Systems kann aber auch von den organisierten Sozialpartnern, Kammern oder von wissenschaftlichen Einrichtungen initiiert werden. Allein die Existenz einer Transparenz- und Orientierungsplattform könnte den strategischen Gestaltungsdialog beleben und fachlich auf eine verbesserte Basis stellen.

7 Der "Arbeitsreport Krankenhaus“ (Bräutigam et al. 2013a) sowie das „Innovationspanel Krankenhäuser" (Blum et al. 2012a) liefern hierfür erste Orientierungen. Weitere empirische Quellen könnten die „BIBB/BAuA-Erwerbstätigenbefragung - Arbeit und Beruf im Wandel, Erwerb und Verwertung beruflicher Qualifikationen" (http://www.bibb.de/de/62622.htm) sowie der "Lohnspiegel“ des WSI (www.lohnspiegel.de) sein.

\section{LITERATUR}

Becker, W./Meifort, B. (2006): Die Systematik der Berufe - Trends und Veränderungen im Berufsfeld Gesundheit, in: Becker, W. (Hrsg.): Ausbildung in den Pflegeberufen. Weichen stellen für die Zukunft in Theorie und Praxis, Schriftenreihe des Bundesinstituts für Berufsbildung, Band 1, Bonn, S. 37-48 Bellmann, L./Grunau, P./Maier, F./Thiele, G. (2013): Struktur der Beschäftigung und Entgeltentwicklung in den Gesundheits- und Pflegeeinrichtungen - 2004 bis 2008, in: Sozialer Fortschritt 62 (3), S. 77-87
Bispinck, R./Dribbusch, H./Öz, F./Stoll, E. (2012): Einkommens- und Arbeitsbedingungen in Pflegeberufen. Eine Analyse auf Basis der WSI-Lohnspiegel-Datenbank, Projekt Lohnspiegel.de, Arbeitspapier 07/2012, Düsseldorf

Bollinger, H./Gerlach, A./Pfadenhauer, M. (Hrsg.) (2008): Gesundheitsberufe im Wandel, Frankfurt a. M.

Bosch, G. (2012): Berufliche Weiterbildung in Deutschland 1969 bis 2010: Entwicklung und Reformoptionen, in: Bothfeld, S./Sesselmeier, W./Bogedan, C. (Hrsg.): Arbeitsmarktpolitik in der sozialen Marktwirtschaft: Vom Arbeitsförderungsgesetz zum Sozialgesetzbuch II und III, Wiesbaden, S. 106-125

Bosch, G./Weinkopf, C. (2011): Arbeitsverhältnisse im Dienstleistungssektor, in: WSI-Mitteilungen 64 (9), S. 439-449, http://www.boeckler.de/wsimit_2011_09_bosch.pdf

Blum, K./ Borchers, U./Evans, M./Löffert, S. (2012a): Innovationspanel 2010/2011: Klinikwirtschaft NRW; Ergebnisse einer Befragung nordrhein-westfälischer Krankenhäuser zu ihren Modernisierungsthemen und -projekten im Jahr 2010/2011, Deutsches Krankenhausinstitut, Düsseldorf

Blum, K./Löffert, S./Offermanns, M./Steffen, P. (2012b): Krankenhausbarometer Umfrage 2012, Deutsches Krankenhausinstitut, Düsseldorf

Bräutigam, C./Evans, M./Hilbert, J./Öz, F. (2013a): Arbeitsreport Krankenhaus, Düsseldorf und Gelsenkirchen (im Erscheinen)

Bräutigam, C./Evans, M./Hilbert, J. (2013b): Berufsbilder im Gesundheitssektor: Vom „Berufebasteln" zur strategischen Berufsbildungspolitik, Friedrich Ebert Stiftung, WISO-Diskurs, Bonn

Bundesinstitut für Berufsbildung (BIBB) (2013): BIBB-Datenreport 2013, Tabellen zum Datenreport zum Berufsbildungsbericht im Internet, Köln Bundesministerium für Familie, Senioren, Frauen und Jugend (BMFSFJ) (2011): Neue Wege - Gleiche Chancen. Gleichstellung von Frauen und Männern im Lebensverlauf, Erster Gleichstellungsbericht, Berlin

Bundesministerium für Wirtschaft und Technologie (BMWi) (2013): Gesundheitswirtschaft: Fakten \& Zahlen, Berlin

Bund-Länder-Arbeitsgruppe zur Weiterentwicklung der Pflegeberufe (2012): Eckpunkte zur Vorbereitung des Entwurfs eines neuen Pflegeberufegesetzes, http://www.bmg.bund.de/fileadmin/dateien/Downloads/P/Pflegeberuf/ 20120301_Endfassung_Eckpunktepapier_Weiterentwicklung_der_Pflegeberufe. pdf (letzter Zugriff: 15.04.2013)

Busemeyer, M. (2009): Wandel trotz Reformstau - Die Politik der beruflichen Bildung seit 1970, Frankfurt a. M./New York

Dahme, H.-J./Kühnlein, G./Stefaniak, A./Wohlfahrt, N. (2012): Leiharbeit und Ausgliederung in diakonischen Sozialunternehmen. Der "Dritte Weg" zwischen normativem Anspruch und sozialwirtschaftlicher Realität. Abschlussbericht eines Forschungsprojektes im Auftrag der Hans-Böckler-Stiftung, Düsseldorf Deutscher Industrie- und Handelskammertag (DIHK) (2013): 10 wichtige Punkte zur Gesundheitswirtschaft für einen Koalitionsvertrag aus Sicht des DIHK, Berlin Deutsche Krankenhausgesellschaft (DKG) (2013): Foliensatz Krankenhausstatistik, http://www.dkgev.de/media/file/13108.Foliensatz_Krankenhausstatistik_20130123.pdf

Dielmann, G. (2013): Krankenpflegegesetz und Ausbildungs- und Prüfungsverordnung für die Berufe in der Krankenpflege, Kommentar für die Praxis, Frankfurt a. M.

Deutsches Krankenhausinstitut (DKI) (2012): Krankenhaus Barometer,

Deutsches Krankenhausinstitut, Düsseldorf

Dunkel, W./Weihrich, M. (Hrsg.) (2012): Interaktive Arbeit. Theorie, Praxis und Gestaltung von Dienstleistungsbeziehungen, Wiesbaden

Esser, F. H. (2012): Im System bleiben - auch bei den Gesundheitsberufen!, in: BWP-Berufsbildung in Wissenschaft und Praxis 41 (6), S. 3

Euler, D. (2013): Das duale System in Deutschland - Vorbild für einen Transfer ins Ausland?: Bertelsmann Stiftung, Gütersloh

Evans, M/Hilbert, J./Kluska, D./Öz, F. (2012): Scheitert die Zukunft der Gesundheit an der Arbeit? Empirische Befunde zur Bewertung des Arbeitsalltags in den Gesundheitsberufen und ihre Bedeutung für die Arbeitsgestaltung, in: Bovelet, J./Holzgreve, A. (Hrsg.): Klinik, Struktur, Versorgung: Medizinisch Wissenschaftliche Verlagsgesellschaft, Berlin, S. 63-81

Evans, M./Galtschenko, V./Hilbert, J. (2013): Befund Sociosclerose. Sozialwirtschaft in der Interessensblockade?, in: Sozialer Fortschritt 62 (8-9),

S. $209-216$

Friedrich Ebert Stiftung (FES) (Hrsg.) (2010): Perspektiven der Erwerbsarbeit: Facharbeit in Deutschland: Friedrich Ebert Stiftung, WISO-Diskurs, Bonn

Gerlinger, T. (2013): Wettbewerb und Privatisierung: Über den Wandel von Gesundheitssystemen, Hamburg

Goldschmidt, A./Hilbert, J. (Hrsg.) (2009): Gesundheitswirtschaft in Deutschland: Die Zukunftsbranche, Stuttgart 
Höppner, K./Kuhlmey, A. (2009): Gesundheitsberufe im Wandel - Relation von ärztlichen und nicht ärztlichen Berufsgruppen, in: Gesundheit Gesellschaft Wissenschaft (GGW) 9 (2), S. 7-14

Isfort, M./Neuhaus, A./Brühe, R. (2012): Landesberichterstattung Gesundheitsberufe Nordrhein-Westfalen 2011, Ministerium für Gesundheit, Emanzipation, Pflege und Alter NRW, Düsseldorf

Klaes, L./Köhler, T./Rommel, A./Schüler, G./Schröder, H. (2013): Public Private Health - Neue Qualifikationsanforderungen in der Gesundheitswirtschaft, Bielefeld

Lohmann, H./Preusker, U. (Hrsg.) (2013): Gesundheitswirtschaftspolitik: Frischer Wind durch neues Denken, Heidelberg, S. 17-38

Mühlbauer, B./ Kellerhoff, F./Matusiewicz, D. (Hrsg.) (2012): Zukunftsperspektiven der Gesundheitswirtschaft, Münster

Oschmiansky, H. (2013): Zwischen Professionalisierung und Prekarisierung: Altenpflege im wohlfahrtsstaatlichen Wandel in Deutschland und Schweden, Dissertation an der Freien Universität Berlin (Elektronische Ressource), Berlin

Streeck, W./Hilbert, J./Kevelaer, K.-H. von/Maier, F./Weber, H. (1987): Steuerung und Regulierung der beruflichen Bildung. Die Rolle der Sozialpartner in der Ausbildung und beruflichen Weiterbildung in der Bundesrepublik Deutschland, Berlin

Voss-Dahm, D./Bosch, G./Mühge, G./Schmierl, K./Struck, O. (2011): Stützen für qualifizierte Facharbeit: Ansätze zum Ausgleich von Stabilität und Flexibilität im Betrieb und auf dem Arbeitsmarkt, in: Voss-Dahm, D./Mühge, G./ Schmierl, K./Struck, O. (Hrsg.): Qualifizierte Facharbeit im Spannungsfeld von Flexibilität und Stabilität, Wiesbaden, S. 7-21

Wissenschaftsrat (WR) (2012): „Empfehlungen zu hochschulischen Qualifikationen für das Gesundheitswesen", WR Drs. 2411-12, Berlin

\section{AUTOREN}

JOSEF HILBERT, Privatdozent, Dr., Soziologe und Gesundheitsökonom, Geschäftsführender Direktor am Institut Arbeit und Technik (IAT) der Westfälischen Hochschule, Gelsenkirchen. Arbeitsschwerpunkte:Trendmonitoring in der Gesundheitswirtschaft, Alternde Gesellschaften, Gesundheitswirtschaft und Strukturpolitik.

hilbert@iat.eu

CHRISTOPH BRÄUTIGAM, Pflegewissenschaftler, wissenschaftlicher Mitarbeiter am IAT, Gelsenkirchen. Arbeitsschwerpunkte: Arbeit und Organisation in Krankenhäusern und Pflegeeinrichtungen, Genderfragen in Krankenhäusern, Professionalisierung der Pflege, intra- und interprofessionelle Kooperation.

braeutigam@iat.eu

MICHAELA EVANS, Sozialwissenschaftlerin, Projektgruppenleiterin „Arbeit und Qualifizierung" am IAT, Gelsenkirchen. Arbeitsschwerpunkte: Arbeit und Qualifizierung in der Gesundheits- und Sozialwirtschaft; Steuerung, Regulierung, Arbeitgeberorganisationen, Gewerkschaften und Verbände in der Gesundheits- und Sozialwirtschaft.

evans@iat.eu 